\title{
The study of the mixing layer particles distribution in dependence on the convective Mach numbers
}

\begin{abstract}
The numerical studies of the quasi twodimensional supersonic turbulent gas-particle mixing layer are performed using the 2D-DNS (Direct Numerical Simulations). The system of Navier-Stokes equations of a multi-species flow is solved using the ENO scheme of a third-order accuracy. The dispersion of the particles is simulated by the Lagrangian method following their trajectories in the mixing layer. The study focuses on the roles of the large-scale vortex structures in a particle dispersion dependence on the Stokes numbers and convective Mach numbers.
\end{abstract}

Altyn Makasheva, Altynshash Naimanova

Keywords - mixing layer, convective Mach number, particle dispersion, 2D-DNS, multi-species flow.

\section{INTRODUCTION}

$\mathrm{T}_{\mathrm{t}}$

URBULENT flows with particle apply in numerous technological applications. These include many propulsion and an energy conversion systems such as processes like combustion of solid and liquid fuels, distribution of pollutants in the atmosphere. For example, in the jet engines, some of the combustion products are released in the solid state.

The numerous studies show that the main characteristics of a turbulent mixing layers strongly depend on the Mach number and the Stokes number [1-4]. Also the compressibility effects play an important role in determining the behavior of the mixing layers and the disperse of the particles.

The numerical simulation of the particle dispersion in the mixing layer is the most preferable in a view of a high complexity and costliness of experiments. In the computational study of the two phase flow there are two approaches. In the first method the discrete elements are tracked through a continuous ?uid by solving system of an ordinary equations of each elements. This is the EulerianLagrangian aproach. In the second methodology, both phases are explored as two interpenetrating continuums and are governed by a set of a differenrial equations representing the conservation laws; this approach is named the EulerianEulerian.

A. A. Makasheva and A. Naimanova are with the Institute of Mathematical Modeling, Almaty, Kazakhstan (corresponding author to provide phone: 8 (727)72-00-48; e-mail: altyn-mak@mail.ru).
The influence of the large-scale turbulent motion of the planar mixing layer on the particle dispersion and their correlation have been shown in [5] using the EulerianEulerian approaches, where has been studied the dispersion mechanisms of the small water particles in the turbulent mixing layer. Hishida et al. [6] investigated the dispersion of the solid particles in a planar shear layer by the EulerianLagrangian methods and observed a strong correlation between the particle dispersion and the Stokes number, defined as the ratio of the particle response time to the characteristic time of the large-scale eddies. The vortex structures in the spatially-developing shear layer of the particle dynamics is numerically studied in [7-9]. There, the investigation has focused on the effects that develope structures on particle dispersion of the different size in the shear layer. It has been indicated that the particle for small and moderate sizes $\left(0<d_{p}<20 \mathrm{pm}\right)$ have been captured in the vertical structures and the larger particles remain relatively unaffected by the large structures. Another important observation was that the particles injected in the fast stream, have dispersed more than those injected in the slow stream. In [10] have been investigated the mechanisms of the particle dispersion in the spatial mixing layer. It has been revealed that the number of the particles moving from the upper stream into the lower is larger than that moving in the opposite direction. The effects of gravity on the particle transport and distribution have also been investigated.

The numerical investigation of the dynamics of the heavy particles in a vortices has been presented in [11]. In there the effects of vortices on the dispersion of the particles have been studied, and only has been obtained that the very low Stokes number particles have accumulated at the vortex center.

In [12] has been made the comparing of the two modeling methods (Eulerian and Lagrangian models) and the numerical results have been compared with the experimental data. The comparison has shown that both of the methods could well predict the steady-state particle concentration distribution. In the unsteady case, the Lagrangian method has shown more realistic behavior of the particles than the Eulerian method.

The objective of the present study is the particle dispersion in the quasi-2D developing compressible free mixing layer. The inflow profile of physical parameters across the hydrogen-nitrogen and air flows is assumed to vary smoothly according to a hyperbolic-tangent function (Fig. 1). At the 
entrance, the solid particles are injected with randomly size to the mixing layer.

Mathematical formulation of the problem is based on the unsteady compressible Navier-Stokes equations of a multispecies flow and the Lagrangian approach to trace the particles. The investigation focuses on the influence of a spatially developing vortex structures on a particle dispersion in a transitional free shear layer depending on the particle size and convective Mach numbers.

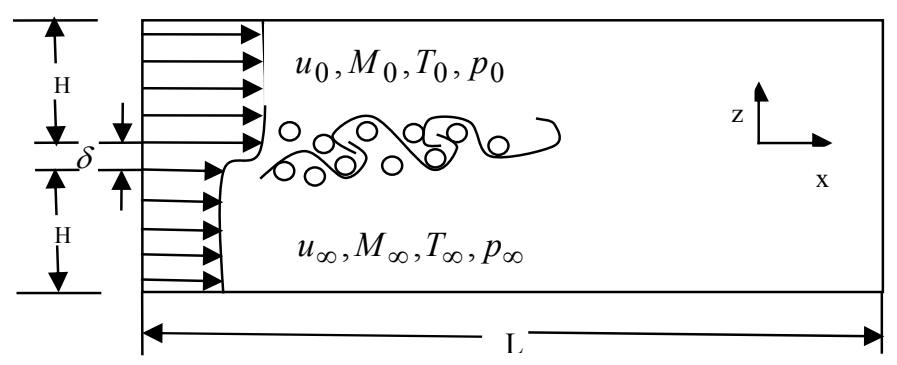

Fig. 1 illustration the flow configuration

\section{THE MATHEMATICAL MODEL AND GOVERNING EQUATIONS}

\section{A. Euler Equation For Multi-Species Gas}

The time-dependent, compressible two-dimensional (2D) planar system of the Navier-Stokes equations for multi-species gas is written in the conservation vector form as:

$$
\frac{\partial \vec{U}}{\partial t}+\frac{\partial\left(\vec{E}-\vec{E}_{v}\right)}{\partial x}+\frac{\partial\left(\vec{F}-\vec{F}_{v}\right)}{\partial z}=0
$$

where the vector of the unknown variables and the vector fluxes are defined by

$$
\begin{aligned}
& \vec{U}=\left(\rho, \rho u, \rho w, E_{t} \rho Y_{k}\right)^{T}, \\
& \vec{E}=\left(\rho u, \rho u^{2}+p, \rho u w,\left(E_{t}+p\right) u, \rho u Y_{k}\right)^{T}, \\
& \vec{F}=\left(\rho w, \rho u w, \rho w^{2}+p,\left(E_{t}+p\right) w, \rho w Y_{k}\right)^{T}, \\
& \vec{E}_{v}=\left(0, \tau_{x x}, \tau_{x z}, u \tau_{x x}+w \tau_{x z}-q_{x}, J_{k x}\right)^{T}, \\
& \vec{F}_{v}=\left(0, \tau_{x z}, \tau_{z z}, u \tau_{x z}+w \tau_{z z}-q_{z}, J_{k z}\right)^{T} .
\end{aligned}
$$

The components of the viscous stress tensor are given as $\tau_{x x}=\frac{\mu}{\operatorname{Re}}\left(2 u_{x}-\frac{2}{3}\left(u_{x}+w_{z}\right)\right) ; \quad \tau_{z z}=\frac{\mu}{\operatorname{Re}}\left(2 w_{z}-\frac{2}{3}\left(u_{x}+w_{z}\right)\right) ;$ $\tau_{x z}=\tau_{z x}=\frac{\mu}{R e}\left(u_{z}+w_{x}\right) ;$

The heat flux is defined by
$q_{x}=\frac{1}{\operatorname{Pr} \operatorname{Re}}\left(\mu+\frac{\mu}{\sigma_{k}}\right) \frac{\partial T}{\partial x} ; \quad q_{z}=\frac{1}{\operatorname{Pr} \operatorname{Re}}\left(\mu+\frac{\mu}{\sigma_{k}}\right) \frac{\partial T}{\partial z} ;$

and the diffusion flux is determined by

$J_{k x}=-\frac{\mu_{l}}{S c \operatorname{Re}} \frac{\partial Y_{k}}{\partial x}, \quad J_{k z}=-\frac{\mu_{l}}{S c \operatorname{Re}} \frac{\partial Y_{k}}{\partial z}$.

where $Y_{k}$ is the mass fraction of $k^{\text {th }}$ species, $k=1 \ldots N$, where $N$ is the number of components in a gas mixture. $\tau, q$ and $J_{k}$ are the viscous stress tensor, the heat flux and the diffusion flux, respectively.

Pressure, total energy and specific entalphy of the $k^{\text {th }}$ species are defined by

$$
p=\frac{\rho T}{\gamma_{\infty} M_{\infty}^{2}}\left(\sum_{k=1}^{N} \frac{Y_{k}}{W_{k}}\right), \quad E_{t}=\frac{\rho h}{\gamma_{\infty} M_{\infty}^{2}}-p+\frac{1}{2} \rho\left(u^{2}+w^{2}\right)
$$

The specific enthalpy and the specific heat at constant pressure of the $k^{\text {th }}$ species are

$$
h=\sum_{k=1}^{N} Y_{k} h_{k}, \quad h_{k}=h_{k}^{0}+\int_{T_{0}}^{T} c_{p k} d T
$$

The specific heat at constant pressure for each component $c_{p k}$ is:

$$
c_{p k}=C_{p k} / W, \quad C_{p k}=\sum_{i=1}^{5} \bar{a}_{k i} T^{(i-1)}, \quad \bar{a}_{j k}=a_{j k} T_{\infty}{ }^{j-1}
$$

where the molar specific heat $C_{p k}$ is given in terms of the fourth degree polynomial with respect to the temperature, consistent with the JANAF Thermochemical Tables [13].

The system of the equations (1) is written in the conservative, dimensionless form. The air flow parameters are $\rho_{\infty}, u_{\infty}, w_{\infty}, T_{\infty}$, hydrogen jet parameters are $\rho_{0}, u_{0}, w_{0}, T_{0}$. In terms of dimensionless variables, $\rho_{\infty} u_{\infty}^{2}$ is the scale, $R_{0} T_{\infty} / W_{\infty}$ is the enthalpy scale, $R_{0}$ is the molar specific heat scale and $\delta$ (the thickness of the splitter plate) is the spatial distance.

\section{B. Particle Equation in the Lagrangian Frame}

The Lagrangian particle equations (sphere shapes) for the position and the velocity are simulated by the system of the ordinary differential equations: 


$$
\begin{aligned}
& \frac{\partial}{\partial t} \vec{x}_{p}=\vec{u}_{p} \\
& \frac{\partial}{\partial t} \vec{u}_{p}=D_{p}\left(\vec{u}-\vec{u}_{p}\right)
\end{aligned}
$$

where $\vec{x}_{p}$ and $\vec{u}_{p}$ are respectively the position and the velocity vectors for a particle represented by the subscript $p$; $D_{p}$ is the drag force with the particle radius $r_{p}$ is given by

$$
D_{p}=\frac{3}{8} \frac{\rho}{\rho_{p}} \frac{\left|\vec{u}-\vec{u}_{p}\right|}{r_{p}} C_{D}\left(R e_{p}\right)
$$

The drag coefficient $C_{D}$ is taken in accordance with the solid sphere drag correlation [14]:

$$
C_{D}= \begin{cases}\frac{24}{\operatorname{Re}_{p}}\left(1+\frac{1}{6} R e_{p}^{2 / 3}\right), & R e_{p} \leq 1000 \\ 0,424, & R e_{p}>1000\end{cases}
$$

and $R e_{p}=\frac{2 \rho\left|\vec{u}-\vec{u}_{p}\right| \cdot r_{p}}{\mu}$ is the particle Reynolds number, $\mu$ is the gas viscosity.

The equation (2) with the force of Stokes viscous drag is written as follows:

$$
\frac{\partial}{\partial t} \vec{u}_{p}=\frac{f_{p}}{S t}\left(\vec{u}-\vec{u}_{p}\right)
$$

where $f_{p}=1+\frac{1}{6} \operatorname{Re}_{p}^{2 / 3}$ is the modification factor for the Stokes drag coefficient, $S t=\frac{\rho_{p} d_{p} \operatorname{Re}_{p}}{18 \rho}$.

The equation for the particle energy is described by the following

$$
\begin{aligned}
& m_{p} C_{p} \frac{d T_{p}}{d t}=2 \pi r_{p} K_{c o n v}\left(T-T_{p}\right) N u_{p} \\
& \rho_{p}=m_{p} /\left(\frac{4}{3} \pi r_{p}^{3}\right)
\end{aligned}
$$

where ${ }^{m} p$ is the particle mass, $\rho_{p}$ is the density of the solid particles, $K_{c o n v}=\left(\mu c_{p}\right) / \operatorname{Pr}_{p}$ is the convective heat transfer coefficient between the gas and the particle.

\section{INITIAL AND BOUNDARY CONDITIONS}

At the entrance:

- for multi-species gas:

$$
u=M_{0} \sqrt{\frac{\gamma_{0} R_{0} T_{0}}{W_{0}}}, w=0, p=p_{0}, T=T_{0}, Y_{k}=Y_{k 0}
$$

at $x=0, \quad 0 \leq z<\mathrm{H}_{1}$.

$$
\begin{aligned}
& u=M_{\infty} \sqrt{\frac{\gamma_{\infty} R_{0} T_{\infty}}{W_{\infty}}},{ }^{w}=0, p=p_{\infty}, T=T_{\infty}, Y_{k}=Y_{k \infty} \\
& \text { at } x=0, \quad \mathrm{H}_{1}+\delta \leq z \leq \mathrm{H}_{2} .
\end{aligned}
$$

In the region of $\mathrm{H}_{1} \leq z \leq \mathrm{H}_{1}+\delta$ all physical variables are varied smoothly from the hydrogen (fuel) flow to the air flow using a hyperbolic-tangent function of any variable $\phi$, so the inflow profiles are defined by

$$
\begin{aligned}
& \quad \phi(\mathrm{z})=0.5\left(\phi_{2}+\phi_{1}\right)+0.5\left(\phi_{2}-\phi_{1}\right) \tanh \left(0.5 z / \delta_{\theta}\right) \text { at } x=0, \\
& 0 \leq z \leq H .
\end{aligned}
$$

where $\phi=\left(u, v, p, T, Y_{k}\right), \quad \delta_{\theta}$ is the momentum thickness. The pressure is assumed to be uniform across the mixing layer. On the lower and upper boundaries the condition of symmetry is imposed. At the outflow, the non-reflecting boundary condition is used [15].

In order to produce the roll-up and pairing of vortex rings, an unsteady boundary condition for velocity field is used at the inlet plane [16], i.e.

$$
\begin{aligned}
& u=\Delta U \cdot \text { Gaussian } \cdot \sum_{m=0}^{3} A \cdot \cos \left(\omega \cdot t+\phi_{m}\right) \\
& w=\Delta w_{\text {factor }} \cdot \Delta U \cdot \text { Gaussian } \cdot \sum_{m=0}^{3} A \cdot \sin \left(\omega \cdot t+\phi_{m}\right)
\end{aligned}
$$

$\operatorname{Gaussian}(z)=\exp \left(-z^{2} / 2 \sigma^{2}\right), \quad \omega=\left(a_{0}+a_{\infty}\right) /\left(2 \delta_{\theta}\right)$

The random phase equation has the following form

$$
\phi=\phi+\operatorname{sign}(\Delta \phi, \text { random }), \quad-1 \leq \text { random } \leq 1,
$$

where $\Delta U=\left(u_{\infty}-u_{0}\right)$ is the difference of the two stream velocities, which measures the strength of shearing. Gaussian $(z)$ is the Gaussian function which has a peak value of a unity at $\mathrm{z}=0$ and the $\pm 2 \sigma$ width is matched to the vorticity layer thickness at the entrance. Coefficient $A=0.001$ is the forcing amplitude. The $\Delta w_{\text {factor }}$ is taken as [15]. The $\omega$ is the excitation frequency, $\delta_{w}=\frac{\left(u_{\infty}-u_{0}\right)}{(\partial u / \partial z)_{\max }}$ is the vorticity thickness, $a_{0}, a_{\infty}$ - sound velocities. 


\section{METHOD OF SOLUTION}

The numerical solution of 2D-DNS equations are calculated in two steps. The first-step solves for the gas dynamic parameters $\left(\rho, u, w, E_{t}\right)$ and second-step the species $\left(Y_{k}, k=1, \ldots, 7\right)$ with mass source terms. The approximation of convection terms are performed by the ENO-scheme of third-order accuracy [17]. The ENO scheme is constructed on the basis of Godunov method, where piecewise polynomial function is defined by the Newton's formula of the third degree. In approximation of derivatives of diffusion terms, second-order central-difference operators are used. The system of the finite difference equations are solved by using matrix sweep method. Then it is necessary to define Jacobian matrix which in the case of the thermally perfect gas represents difficult task. This problem is connected by explicit representation of pressure through the unknown parameters. Here pressure is determined by using the following formula $[17,18]$

$$
p=(\bar{\gamma}-1)\left[E_{t}-\frac{1}{2} \rho\left(u^{2}+w^{2}\right)-\rho \frac{h_{0}}{\gamma_{\infty} M_{\infty}^{2}}\right]+\frac{\rho T_{0}}{M_{\infty}^{2} W}
$$

where $\bar{\gamma}=h_{s m} / e_{s m}$ is an effective adiabatic parameter of the gas mixture, $\quad h_{s m}=\sum_{i=1}^{N} Y_{i} \int_{T_{0}}^{T} c_{p_{i}} d T \quad e_{s m}=\sum_{i=1}^{N} Y_{i} \int_{T_{0}}^{T} c_{v_{i}} d T$ enthalpy and internal energy of the mixture minus the heat and the energy of formation; $T_{0}=293 \mathrm{~K}$ is the standard temperature of formation. The ordinary equations are solved using the Euler method second order of accuraccy.

\section{RESULTS AND DISCUSSION}

The free shear layer of a hydrogen-air flows mixing with injection of a solid particles is numerically studied. The initial momentum thickness $\delta_{\theta}=9.35 \times 10^{-5} \mathrm{~m}$. At the inflow plane, the hydrogen-nitrogen mixture enters from the upper half: $Y_{H_{2}}=0.5, \quad Y_{N_{2}}=0.5$ and the air $Y_{O_{2}}=0.2$, $Y_{N_{2}}=0.8$ enters from the lower half. The algorithm of the method and the sensitivity of the convergence of solutions to grid characteristics in detailed was in $[17,18]$. In this the 526x201 grid with stretching at the entrance and mixing layer is used. The convective Mach number was taken $M_{c}=\left(u_{c}-u_{\infty}\right) / a_{\infty},\left(u_{c}=\left(a_{\infty} u_{0}+a_{0} u_{\infty}\right) /\left(a_{\infty}+a_{0}\right)\right)$.

Numerical simulation is performed for the following characteristic parameters: $1.5 \leq M_{0} \leq 4,1.5 \leq M_{\infty} \leq 4$ and $0.68 \leq M_{\mathrm{c}} \leq 1.66$, $T_{0}=T_{\infty}=2000 \mathrm{~K}$, $p_{0}=p_{\infty}=101325 \mathrm{~Pa}$. The particles is injected from four input points $x=0, z=20,30,40,50$ with initial conditions:
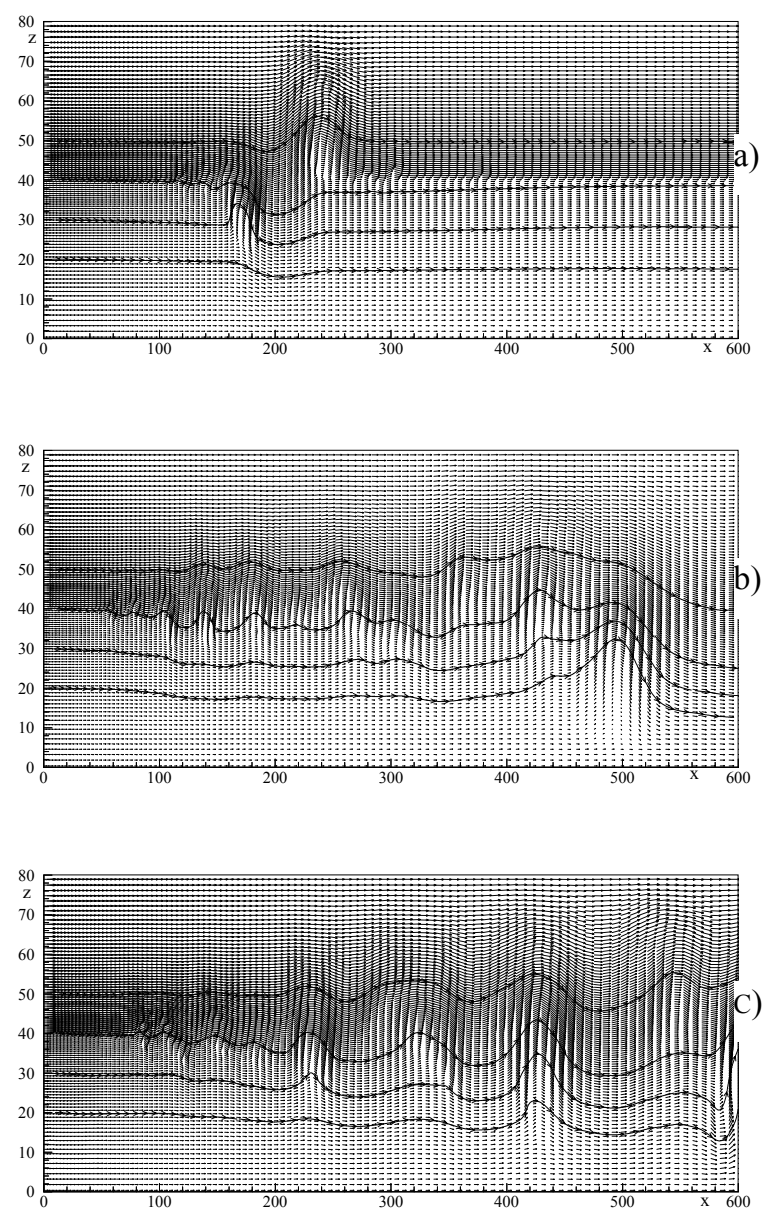

Fig. 2 The distribution of the velocity vector field at time: a) $t=375$, b) $t=1000$, c) $t=1500$ for $d_{p}=150 \mathrm{mkm}$ $M_{0}=2.1, M_{\infty}=2, M_{c}=0.92$

$u_{p 0}=u_{\mathrm{g} 0,} \quad u_{p \infty}=u_{\mathrm{g} \infty}$ and $\rho_{p 0}=\rho_{p \infty}=2560 \mathrm{~kg} / \mathrm{m}^{3}$, the temperature of them at the inlet are assumed to be equal to the values of the air flow. The particles are injected into the flow uniformly, one by one after the period of time $\Delta t=5$, in accordance with [19], where for distribution is used $\mathrm{x}$-squared function is used:

$$
f(r)=\frac{r^{3}}{6 \bar{r}^{4}} e^{-r / \bar{r}}, \bar{r}=\frac{1}{6} d_{p}
$$

here $r$ - injected particles and maximum of it should not exceed the hole nozzles, ${ }^{d} p$ - diameter of the particles. The cumulative distribution function $h(r)$ for the randomly choosen radius of particles is: 


$$
h(r)=1-e^{-r / \bar{r}}\left|1+\frac{r}{\bar{r}}+\frac{1}{2}\left(\frac{r}{\bar{r}}\right)^{2}+\frac{1}{6}\left(\frac{r}{\bar{r}}\right)^{3}\right|
$$

Below are represented the results of a numerical simulation of the problem with following values of the characteristic parameters: $M_{0}=2.1, \quad M_{\infty}=2.0$ with the convective Mach number $M_{c}=1.249$.

In Fig. 2, 3 is showed the sequence of the velocity vector fields (Figure 2) and vorticity contours (Figure 3), which demonstrate the formation of the vortex structures and growth of the mixing layer, in accordance with the mechanism of their formation described in the literatures [19]. Namely, in the
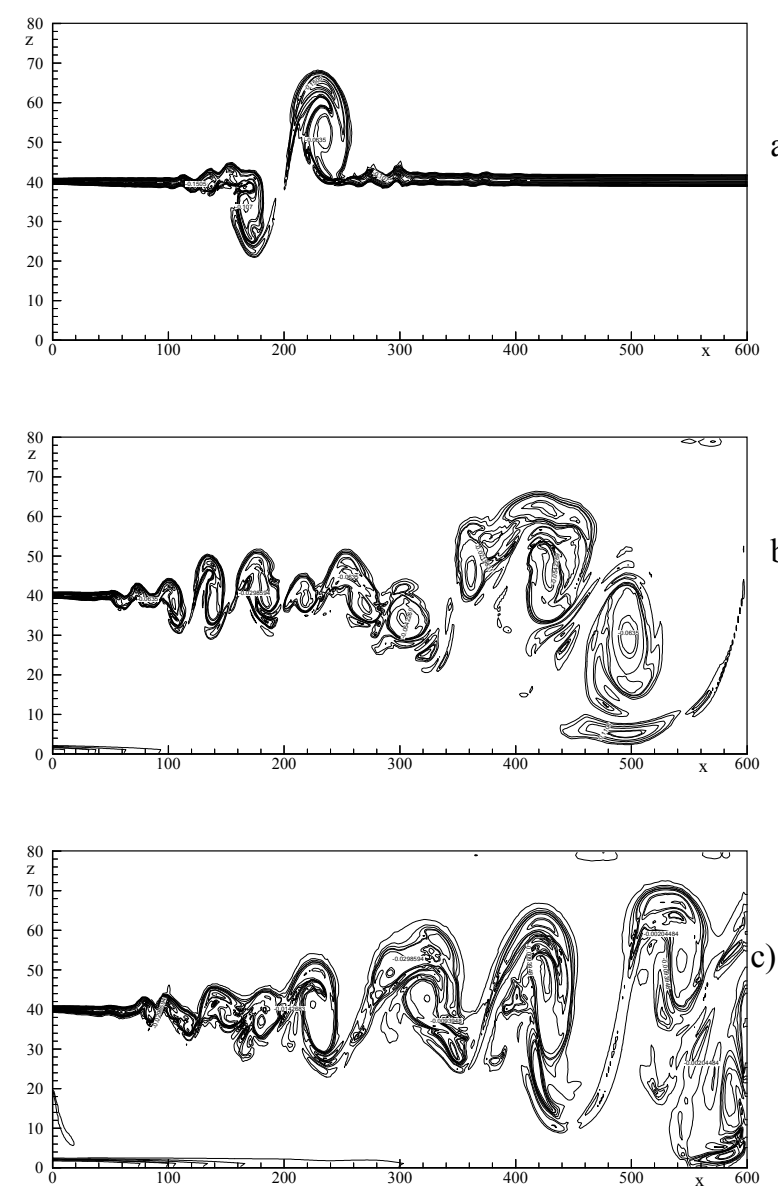

Fig. 3 Distribution of the vorticity isoline at time: a) $t=375$, b)

$$
\begin{gathered}
t=1000, \text { c) } t=1500 \text { for } d_{p}=150 \mathrm{mkm} \\
M_{0}=2.1, M_{\infty}=2, M_{c}=0.92
\end{gathered}
$$

b)
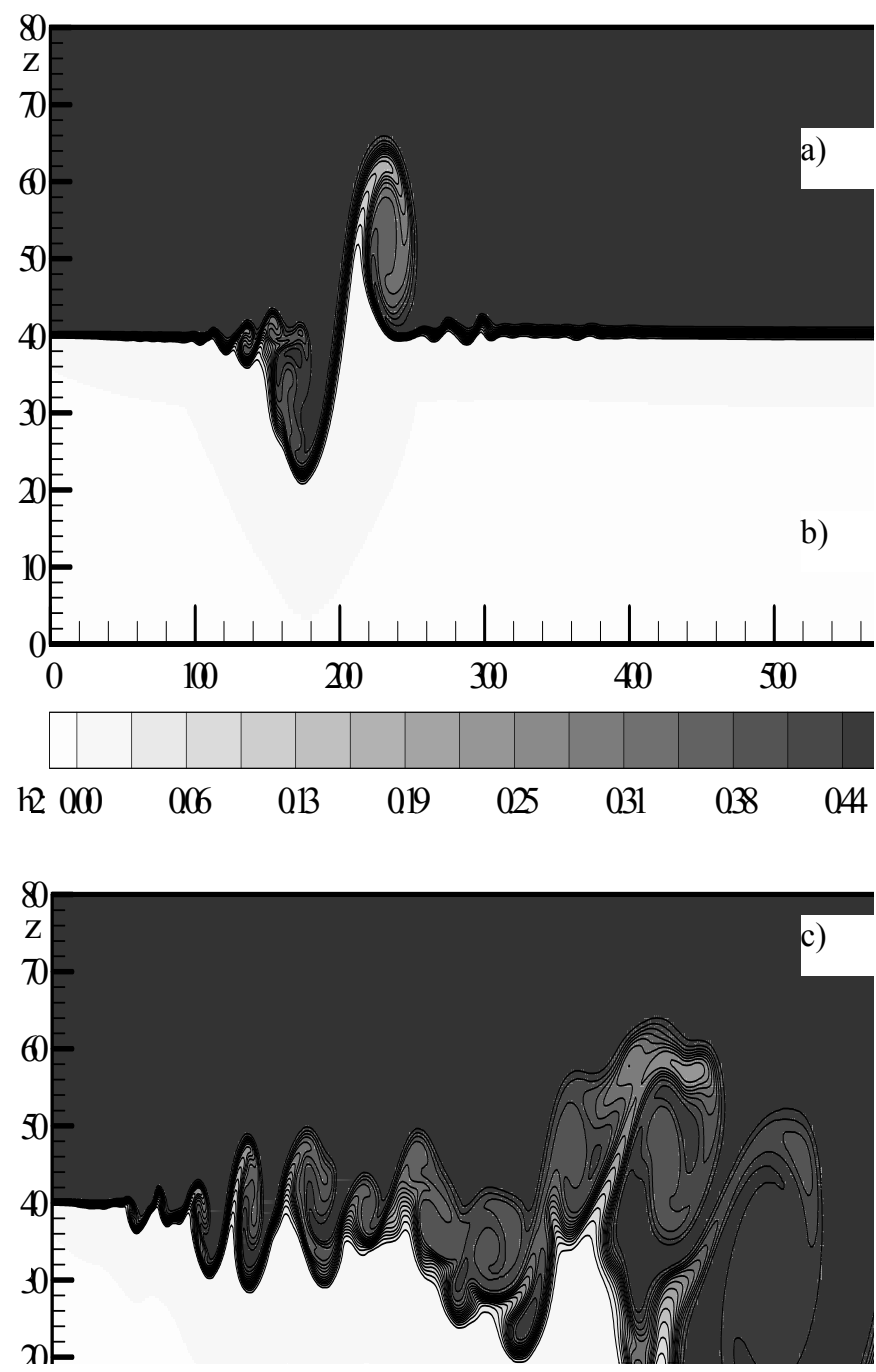

regions of the large velocity gradients $\left(u_{0} / u_{\infty}=2.66\right)$ an instability occurs, as a result of which the roll-up of the vortices is started ( $\mathrm{t}=375, x \approx 200$, Fig. 3a).

The fig. 3,b demonstrates that at a distance from $x=10$ to $x=300$ the vortex system is only enlarging, while from $x=300$ to $x=600$ the neigboring varius are pairing.

The generation of the vorticity are well illustrated by the isolines of hydrogen (Fig. 4,b) too. The formation of the local zones of hydrogen at the centers of the vortices during their pairing is also shown. From the figure $4, \mathrm{~b}$ follows that the air is only formated at the periphery of the large structures.

It is known that the particle movement and their distribution in the mixing layer are influenced by the size of the particles which are characterized by the Stokes numbers (St). In here the injection of the particles of the various size is performed in accordance with $d_{p}=150 \mathrm{mkm}$ and 

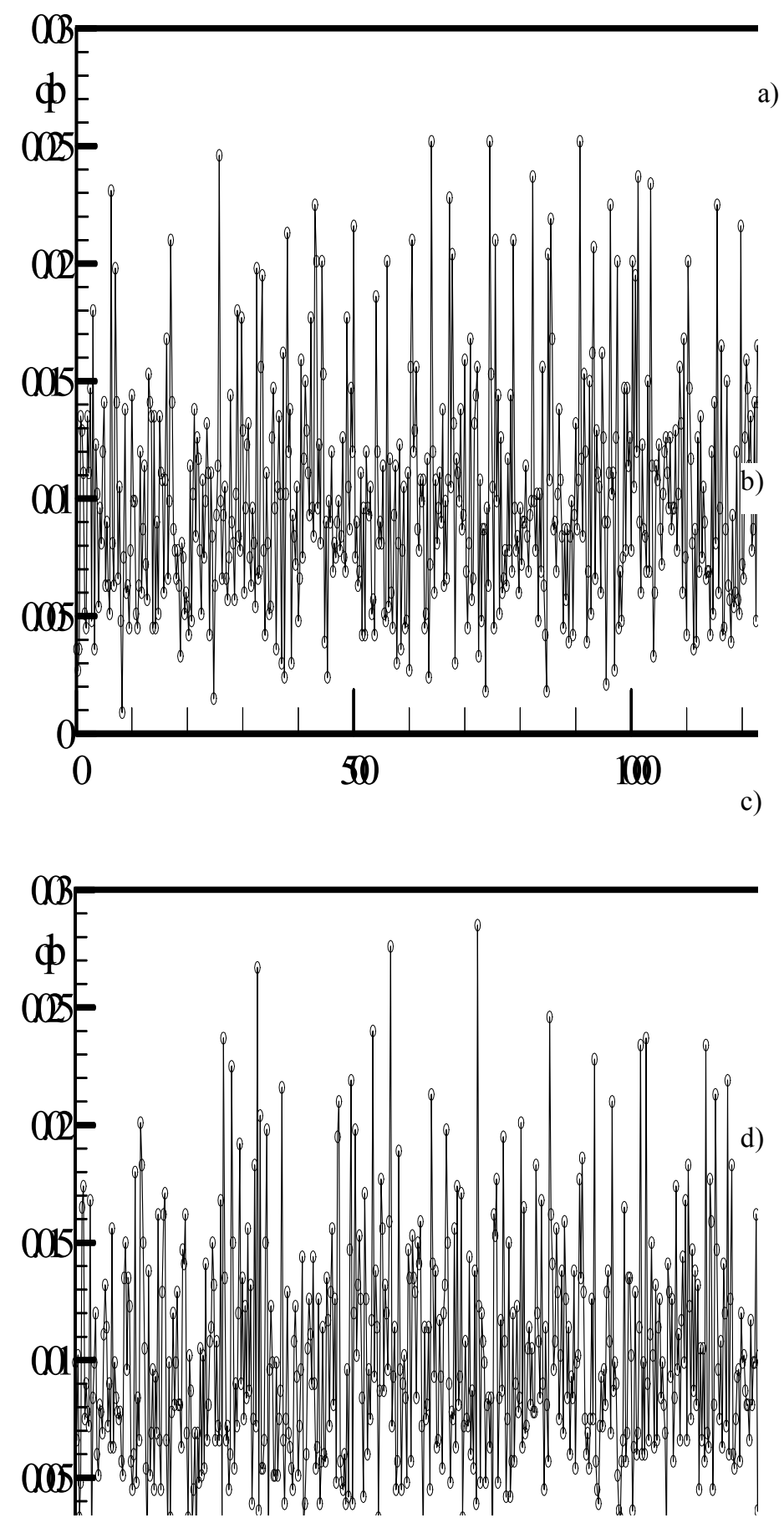

nondimensional $d_{p}=0.0075$ (Figure5: a) $z=20$, b) $z=30$, c) $z=40$, d) $z=50$ ), were the radius of the particles varied in the range from 0.001 to 0.028 . In this case the $\max (f(p))=6.767, \quad \min (f(p))=1.922$; $\max \left(\operatorname{Re}_{p}\right)=183, \quad \min \left(\operatorname{Re}_{p}\right)=13$ and $\max (S t)=0.0095, \min (S t)=0.0062 \quad$ (see the equation (3)). Consequently, $f_{p}>S t$. a)
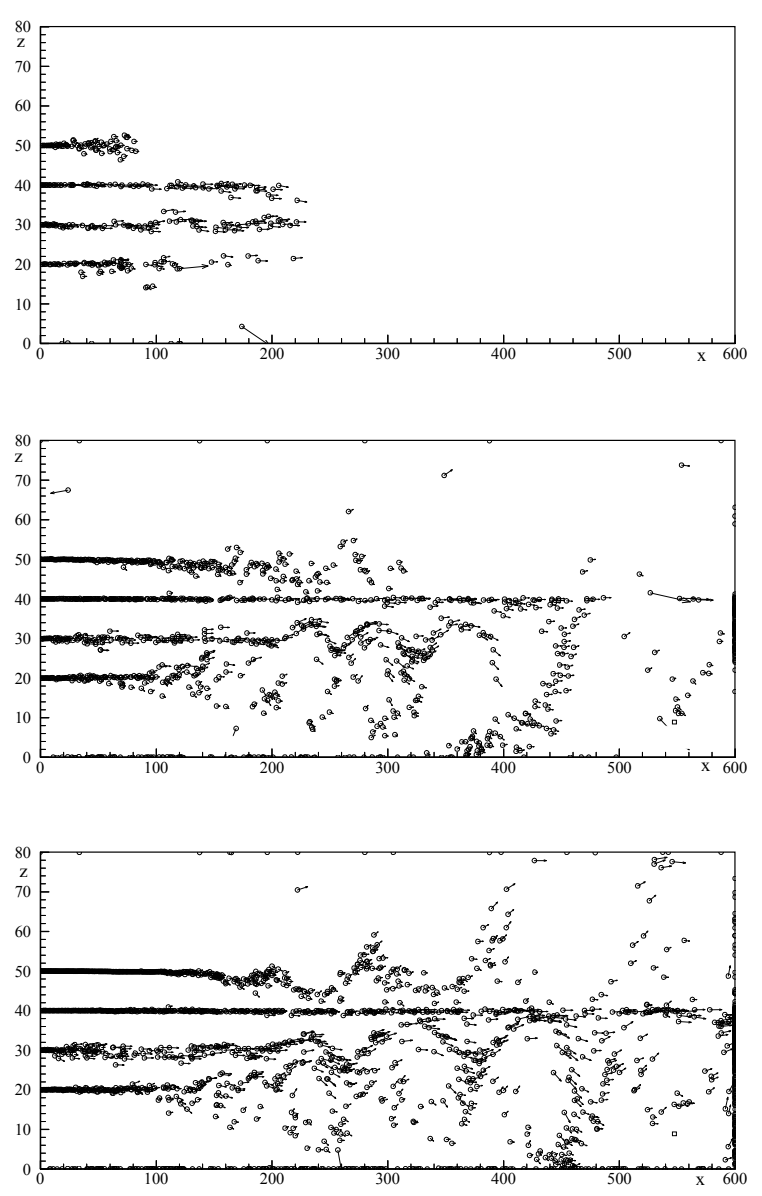

Fig. 6 Particle distribution at time: a) $t=375$, b) $t=1000$, c) $t=1500$ for $d_{p}=150 \mathrm{mkm}$ for $M_{0}=2.1, M_{\infty}=2, M_{c}=0.92$

The corresponding particle dispersion pattern are shown in Figure 6. $\left(f_{p}>S t, S t<1, \operatorname{Re}_{p}>10\right)$. Figure shows the reaction of the particles to the movement of the vortical structures (see fig. 3). For the regions of the low gas velocities $\left(M_{\infty}=2.0\right)$ the picture of the particle dispersion closely resemble the patterns of the flow vortex structures and tend to accumulate around the circumference of the vortex and between vortices with a few number of the solid particles inside of the vortices. And the figure $6 \mathrm{c}$, demonstrates that the particles injected at the entrance $x=0, \quad z=40$ $\left(M_{0}=2.1\right)$ are generally, moved in agreement with proper the trajectory, a namely, the gas flow do not impact on their trajectories.

Figure 7 shows the root mean square of the particle number per cell for each $x$ section through each $\Delta x=4$ at $t=1500$, which is used to quantify the distribution of the particles along the streamwise direction. $\operatorname{Nrms}(\mathrm{x})$ is obtained from: 


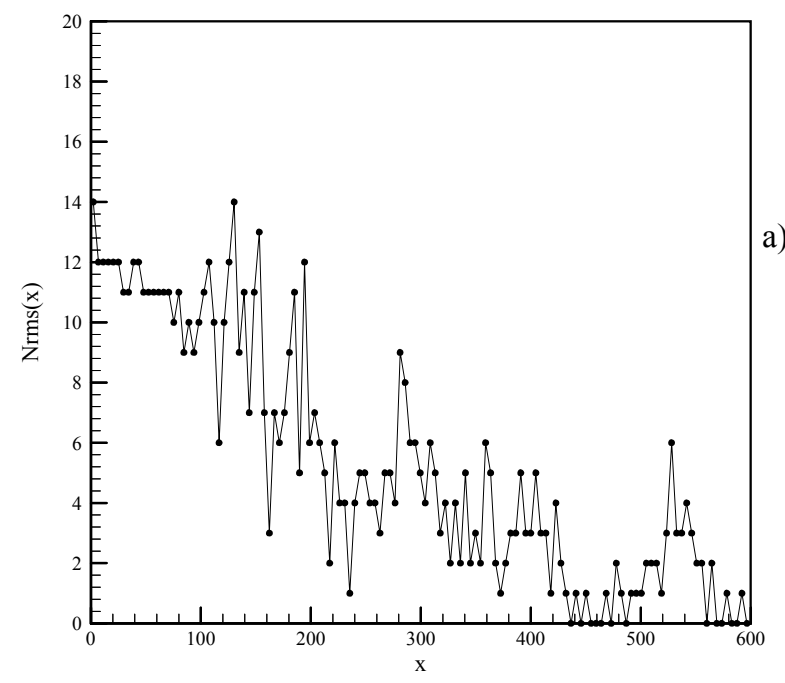

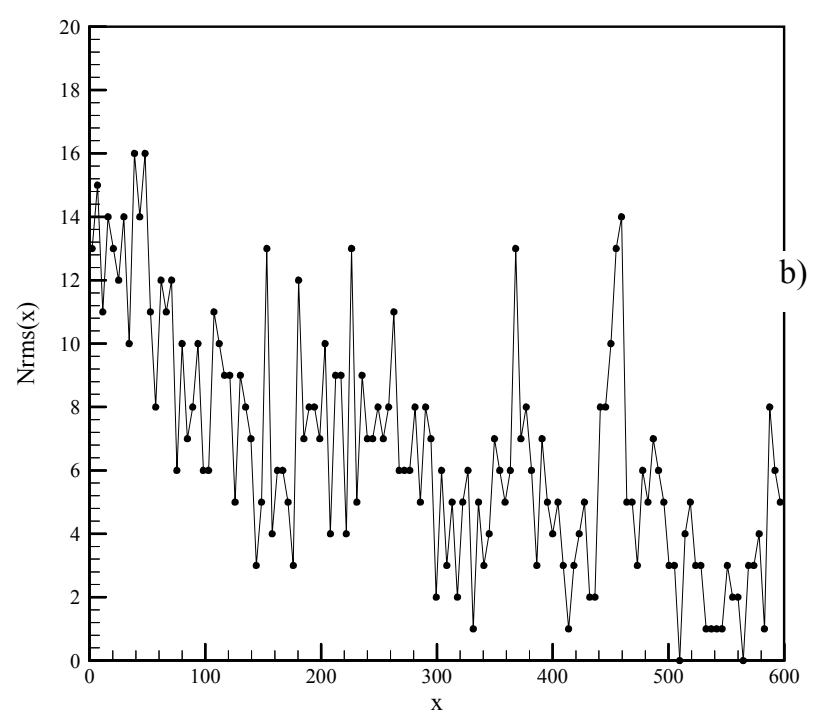

Fig. 7 Quantitative distribution of particles of the upper (a) and lower (b) flows over the cross section $x$ at time $t=1500$ for $d_{p}=150 \mathrm{mkm}$

$$
\begin{gathered}
\text { for } M_{0}=2.1, M_{\infty}=2, M_{c}=0.92 \\
N_{r m s}(x)=\left(\sum_{i=1}^{N_{c p}} \frac{N_{i}(x)^{2}}{N_{c p}}\right)^{1 / 2}
\end{gathered}
$$

where $N_{\mathrm{cp}}$ is the total number of computational cells in one $x$ section and $N_{i}(x)$ is the number of the particles in the $i$ th cell of that $x$ section. The concentration of the particles along the streamwise direction is shown in Figure 7 where the calculation is performed for
0

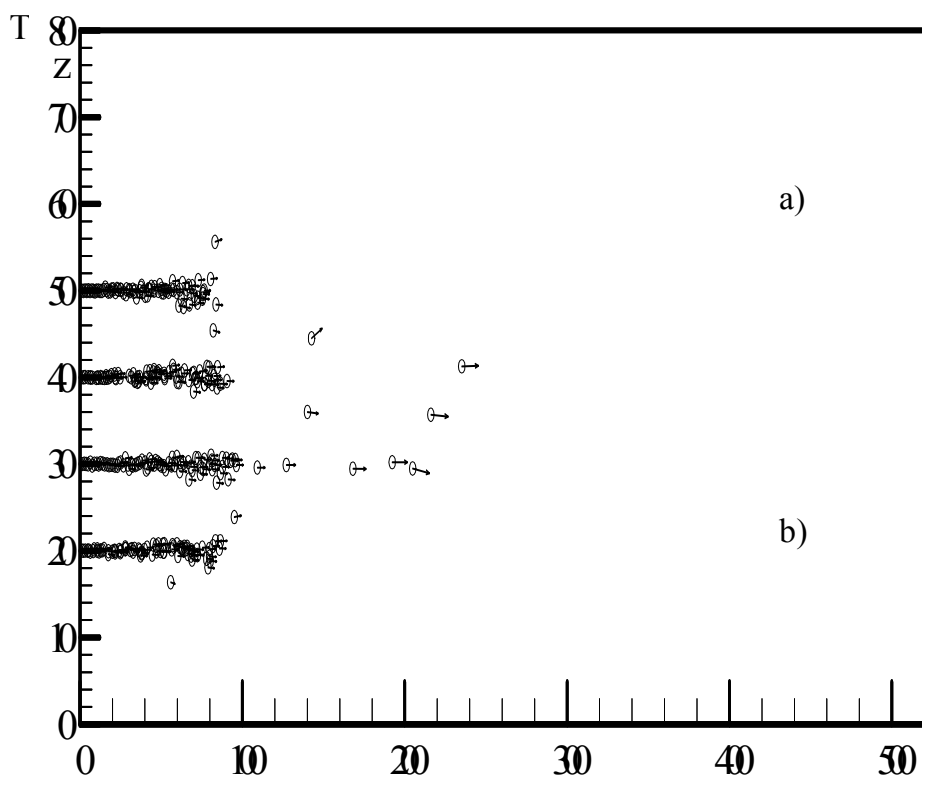

c)

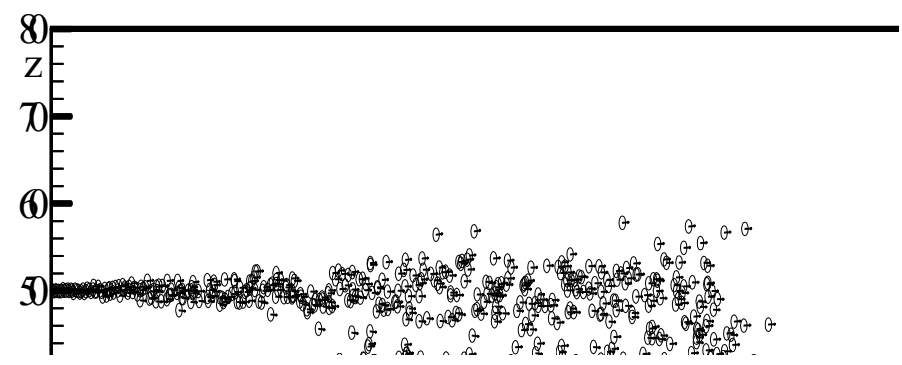

particles are concentrated in the region of trapping of particles by vortices (Fig. 3,c).

Below, it is show the result of the numerical experiments performed for injection of the particles with smaller sizes and $\operatorname{Re}_{p}$. And the particles are injected in accordance with the above pointed distrubation function. The size of the injected particles varies from 0.00001 to 0.00038. In this case $d_{p}=2 \mathrm{mkm}$ (dimensionless $\left.d_{p}=0.0001\right), \quad \max (f(p))=1.3, \quad \min (f(p))=1.05$; $\max \left(\operatorname{Re}_{p}\right)=2.44, \quad \min \left(\operatorname{Re}_{p}\right)=0.17 \quad$ and $\max (S t)=35.45, \min (S t)=7.66$ and $f_{p}<S t$.

Form the figure 8 follows that the particles are nearly unaffected by changes in the gas flow and disperse much less than the flow, for the parameters $f_{p}<S t$, St $>1, \operatorname{Re}_{p}<10$. 
Thus, the obtained results show, that the quality behaviour of the particles do not match with result $[9,10]$. The dispersion of the particles with large sizes is an analogy to the movement of the small particles of the $[9,10]$. Apparently, such divergence is explained by in here $S t<1$ and $f_{p}>S t$, consequently, the force of Stokes is smaller than the Stokes force of $[9,10]$. The reversed behavior is watched for the particles with small sizes.
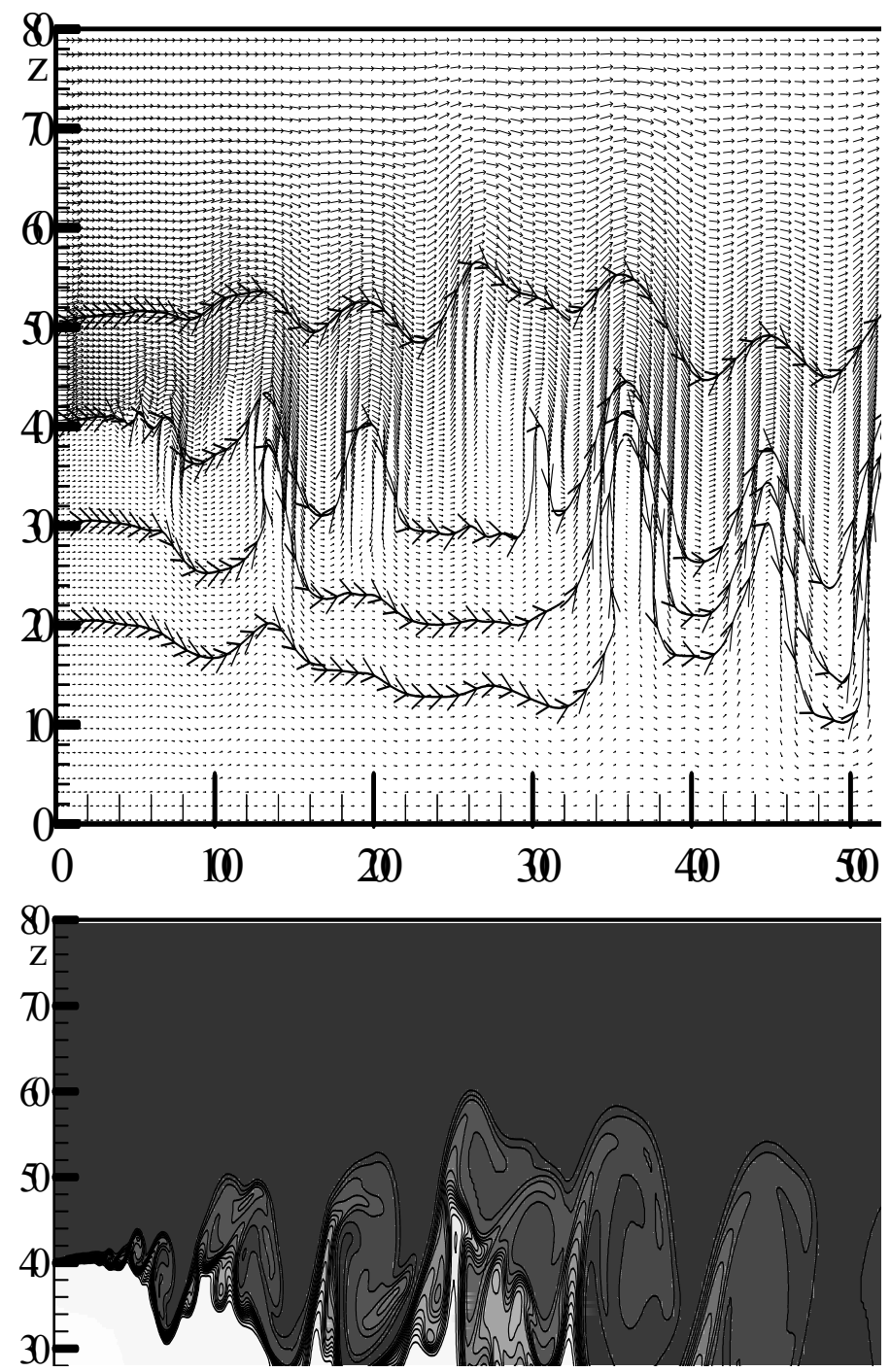

The increase of the Mach number of the hydrogennitrogen mixture (Fig. 9, $M_{0}=3, M_{c}=1.66, d_{p}=150$ $m k m$ ) with fixed $M_{\infty}=2$ leads to the fact that the velocity of the hydrogen is essentially high $u_{0} / u_{\infty}=3.99$ comparing with previous case, respectively, the mixing layer considerably wider (Fig. 9, a, b, $t=1500$ ). For example, figure $4 \mathrm{c}$ shows that the hydrogen is expanded to $z=30$, whereas it is enlarged to $z=40$ (fig. 9, b).
The other picture of the flow is observed for $M_{\infty}=3$, $M_{c}=0.68, u_{0} / u_{\infty}=1.87, d_{p}=150 \mathrm{mkm}, \quad$ for a fixed $M_{0}=2.1$. As follows from Fig. 10, a and Fig. 10, b, the vortical structure is formed symmetrically with respect to the input separation surface (see Fig. 3, c and Fig. 9, c). And additionally the particles are captivated by the vortical structures and curved under the influence of the streamlines of lower gas (Fig. 10, c). As result, the particles, are almost symmetrically involved in the vortex structure.
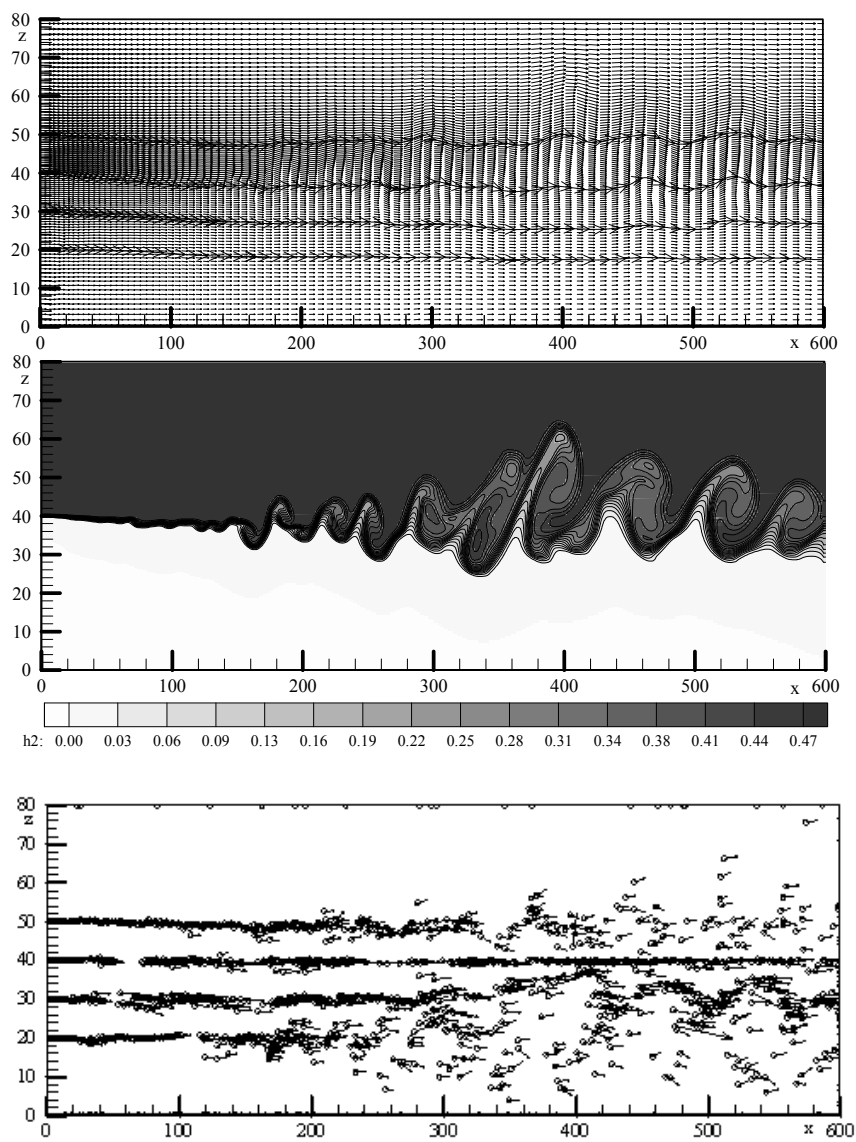

Fig. 10 a) the velocity vector field, b) the mass concentration of hydrogen, c) the particle distribution for $M_{0}=2.1, M_{\infty}=3$, $M_{c}=0.68$ at the time $t=1500$ for $d_{p}=150 \mathrm{mkm}$

\section{EFERENCES}

[1] M. Lesieur, O. Metais, P. Comte "Large-Eddy simulations of turbulence", Cambridge University Press, 2005.

[2] I. Mahle, J. Sesterhenn, R. Friedrich "Turbulent mixing in temporal compressible shear layers involving detailed diffusion processes", J. Turbulence, pp. 1-2, 2007, vol. 8. 
[3] C. Pantano, S. Sarkar "A study of compressible effects in the high-speed turbulent shear layer using direct simulation", J. Fluid Mechanics, pp. 329-371, 2002, vol. 451.

[4] N. Clemens, M. Mungal "Large-scale structure and entrainment in the supersonic mixing layer", J. Fluid Mechanics, pp. 171-216, 1995, vol. 284.

[5] B. J. Lazaro and J. C. Lasheras "Particle dispersion in the developing free layer" Part 2. Forced flow. J. Fluid Mech., pp. 179, 1992, vol. 235.

[6] K. Hishida, A. Ando and M. Maeda "Experiments on particle dispersion in a turbulent mixing layer", J. Multiphase Flow, pp.181, 1992, vol. 18.

[7] G. Jacobs and W. S. Don "A High-Order WENO-Z Finite Difference based Particle-Source-in-Cell Method for Computation of Particle-Laden Flows with Shocks", J. Comp. Phys., pp. 1365-1379, 2009, vol.5

[8] Yiming Li and J. B. McLaughlin "Numerical simulation of particle-laden turbulent channel flow", Physics of fluids, pp. 2957-2967, 2001, vol. 13.

[9] S.K. Aggarwal, J.B. Yapo, F.F. Grinstein, K. Kailasanath "Numerical simulation of particle transport in planar shear layers", Computers \& Fluids, pp. 39-59, 1996, vol. $25,{ }^{1} 1$.

[10] Hu Zhiwei, Luo Xiaoyu, H. Luo Kai "Numerical Simulation of Particle Dispersion in a Spatially Developing Mixing Layer", Theoret. Comput. Fluid Dynamics, pp. 403-420, 2002, vol. 15.

[11] B. Marcu, and E. Meiburg. "The effect of streamwise braid vortices on the particle dispersion in a plane mixing layer. I. Equilibrium points and their stability", Phys. Fluids, pp. 715-733, 1996, vol. 8.

[12] Z. Zhang and Q. Chen "Comparison of the Eulerian and Lagrangian methods for predicting particle transport in enclosed spaces," Atmospheric Environment, pp. 52365248, 2007, vol. 41.

[13] R. Kee "CHEMKIN-II: a Fortran chemical kinetic package for the analysis of gas-phase chemical kinetics", SANDIA,1989.

[14] R. Chein and J.N. Chung "Effects of vortex pairing on particle dispersion in turbulent shear flows", Multiphase Flow, pp. 775-785, 1987, vol.13.

[15] T. Poinsot, S. Lele "Boundary Conditions for Direct Simulation of Compressible Viscous Flows", Computational Physics, pp. 104-129, 1992, vol.101

[16] H. Ounis and G. Ahmadi "Motions of small particles in a turbulent simple shear flow field under microgravity condition" Phys. Fluids A. pp.2559-2570, 1991, vol. 3.

[17] A. Beketaeva, A. Naimanova "Numerical study of spatial supersonic flow of a perfect gas with transverse injection of jets", Applied Mechanics and Technical Physics, pp. 896-904, 2011, vol.52.

[18] P. Bruel, A.Zh. Naimanova "Computation of the normal injection of a hydrogen jet into a supersonic air flow", Thermophysics and Aeromechenics, pp. 531-542, 2010, vol. 17 , No. 4.

[19] A.A. Amsden, P.J. O'Rourke, T.D. Butler “KIVA-II: A computer program for chemically reactive flows with sprays", Los Alamos National Laboratory report, 1989, $158 \mathrm{p}$. 